PACS 73.61.-r

\title{
Charge transport in thin polymer films
}

\author{
R.B. Salikhov ${ }^{\dagger}$ T.R. Salikhov \\ †salikhovrb@ya.ru \\ Bashkir State University, Z.Validi St. 32, 450076 Ufa, Russia
}

In recent years, researchers' attention is drawn not only to the development of an increasing number of different elements of nanoelectronics based on polymer films, but also to the study of electrical properties and the carrier transport mechanisms in these films. This article describes the features of charge transport, including the phenomena at the interface between the polymer and the metal electrode, and in the bulk of the polymer film. The transfer of charge carriers in the polymer layers are mainly determined by the process of hopping between localized molecular states. The simplest form of the hopping conductivity is realized in the transitions between the nearest neighboring centers. The features of the hopping conductivity with a variable hopping and hopping transport in centers with a Gaussian distribution of energy levels were analyzed. The model of polarons and the dependence of the mobility of polarons on the temperature and field were reviewed. It was shown, that, the field dependence of the Poole-Frenkel has a place for both pure charge transfer and the polaron transfer. To describe the charge transport across the border between the polymer and the electrode in sufficiently strong electric fields the models of Fowler-Nordheim tunneling and Richardson-Schottky thermionic emission were used.

Keywords: thermionic emission, hopping transport, polarons.

\section{Introduction}

Electronic conductivity of organic molecular compounds differs from that of metal and inorganic semiconductors such as silicon and germanium. The well-known band theory of crystal lattice is a good base to understand the conduction mechanism of crystalline molecular solids and conjugated and unconjugated polymers. At the same time, the applicability of the ideal elongated chain model to materials with a complicated morphology is naturally limited. Even within the frames of idealized model, the inorganic conductors and semi-conductors differ considerably from polymers. Besides, in polymers the screening of interactions between charge carriers is less; electron-electron and electron-hole interactions play an important role causing considerable localization of electron states as compared with inorganic materials [1]. Absence of macroscopic ordering means inadequacy of band conduction model to describe electron conductivity of bulk polymer materials, though it can be used to a limited extent when studying conduction process.

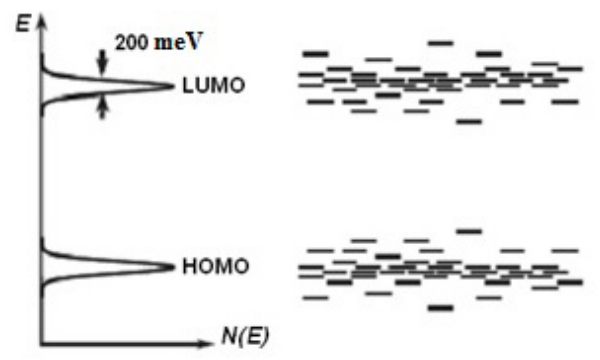

Fig. 1. HOMO and LUMO levels distribution in amorphous organic thin films.
In amorphous layers of thin organic films the terms "conduction band' and "valence band" are usually replaced by the terms of the lowest unoccupied molecular orbital (LUMO) and the highest occupied molecular orbital (HOMO), respectively. The states density is mainly described quite satisfactorily by Gaussian distribution of localized molecular orbitals of individual molecules as it is shown in the fig. 1 [2].

Transport of charge carriers in these amorphous layers is determined primarily by hopping process between localized molecular states. Therefore, the difference between the trap state and regular transport state is not evident. The solution can be found in transport energy concept initially introduced by Monro [3] for amorphous inorganic semi-conductors and later extended for amorphous organic semi-conductors [4, 5]. This concept is based on the following statistic principle: a carrier located in a deep state tail most likely will transfer to the state with energy $E_{t}$ independently of its initial energy in the states' tail (fig.2).

Value $E_{t}$ is called a transport energy or release energy as this value describes the level from which a trapped carrier is released to move to a next center.
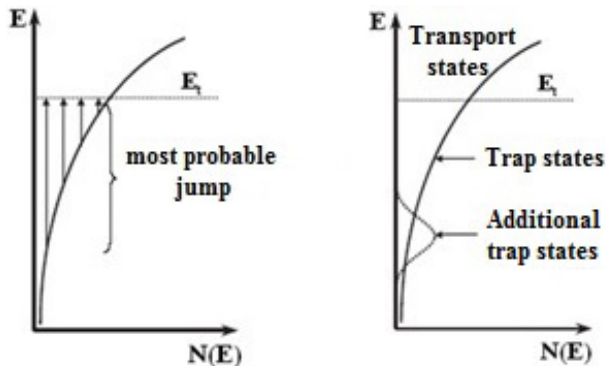

Fig. 2. Transport level of energy $E_{t}$. 
Transport energy has only statistic meaning, but its role is similar to that of band edge or mobility border in inorganic semi-conductors.

Therefore, each state below the transport energy is a trap one while the states above transport energy are the usual transport states though all the states are localized. However, energy of transfer is a function of temperature. A state acting as a trap one at ambient temperature can become a state of transfer at the lower temperatures. In addition ot the trap states located in level distribution tail near HOMO/LUMO, there can be additional trap states at discrete values of energy with arbitrary energy distribution within the values below the transport energy (fig.2).

\section{Charge carrier transportation across the border}

Depending on the size of barrier on the interface of electrode with polymer film electric current flowing through the sample can be of injection type, i.e. limited by space charge.

In this case one of the electrodes should be ohmic one, i.e. it should provide more charge carriers in time unit than the sample is able to transport not breaking the Poisson's law. Otherwise, charge carrier transportation across the interface will be limited by the barrier.

Tunneling model of Fowler-Nordheim (FN) and Richardson-Schottky (RSch) thermionic emission model [6] are usually used to study injection in dielectric in a rather strong electric fields.

A thermal electron emission from hot metal is called thermionic emission. Electrons emission from metal contact into vacuum or dielectric conduction band by their thermal transportation through potential barrier in electric field is called Schottky emission. Taking into account image forces in parabolic approximation it is possible to get RichardsonSchottky equation for current density [6]:

$$
J=A^{*} T^{2} \exp \left[\frac{-e\left(\varphi_{B}-\sqrt{e F / 4 \pi \varepsilon \varepsilon_{0}}\right)}{k T}\right],
$$

where $J$ is a current density, $A^{*}$ is the Richardson constant, $e$ is an electron charge, $\varphi_{B}$ is a barrier height, $F$ is a field density, $\varepsilon$ is a dielectric permeability of a sample, $\varepsilon_{0}$ is the electric constant, $k$ is the Boltzmann constant, $T$ is temperature. The barrier decrease caused by the external field influence amounts to: $\Delta \varphi=\sqrt{\mathrm{eF} / 4 \pi \varepsilon \varepsilon_{0}}$.

An important assumption in RSch model is that electron can be taken out from metal once it gets enough heat energy to cross the potential barrier which is formed by a superimposition of the external field and images forces. Neither tunneling nor inelastic scattering of charge carrier before potential barrier crossing are not considered.

In the electric field between $10^{5} \mathrm{~V} / \mathrm{cm}$ and $2 \cdot 10^{6} \mathrm{~V} / \mathrm{cm}$ barrier decrease in a dielectric with the dielectric constant 3.5 is equal to $0.06-0.28 \mathrm{eV}$, maximum of electrostatic potential is $3-0.7 \mathrm{~nm}$ from the interface [7]. Therefore the barrier height decrease is comparable to the potential barrier height itself, so neglecting the tunneling is not valid.

From the other hand, RSch model usage suffers from a disregard of inelastic scattering of charge carriers which is important for organic substances where transportation is a noncoherent process and mean free path is comparable to the intermolecular distance $1 \mathrm{~nm}$.

According to the quantum theory, electron wave function within dielectric area located between two electrodes is different from zero. Wave function exponentially decreases with a distance into the barrier. If the barrier is very narrow, the probability to pass through the barrier for an electron has a finite value depending on the height and form of the potential barrier. Tunneling (autoemission) can be observed in the case of a wide barrier if its effective thickness decreases under the influence of a strong electric field.

In FN model image forces are disregarded and the tunneling of electrons from metal through a triangle barrier to free states of conduction area is considered. When the field intensity increases, the height and width of potential barrier decreases to such an extent that a new physical effect appears and becomes prevailing: quantum mechanic tunneling of electron across the potential barrier.

Current caused by the tunnel emission facilitated by a field is described by Fowler-Nordheim equation. In this case the current density can be described by the expression [8]:

$$
J(F)=B F^{2} \exp \left(-\frac{4 \sqrt{2 m_{\text {eff }}\left(e \varphi_{B}\right)^{3}}}{3 \hbar \mathrm{F} F},\right.
$$

which is independent from temperature. Here, $m_{\text {eff }}$ is the effective mass of a charge carrier in dielectric, $\hbar$ is the Planck constant.

In spite of disadvantages of both FN and RSch concepts, they have been applied successfully to describe injections of a charge carrier in organic light emitting diodes. E.g., FN model was applied to give reasonable values for the barrier height and to take into account independence of temperature characteristic $J(F)$ in strong fields [9].

It is necessary to mention that electric current values are less than expected one based on equation (2) [10]. It was explained by the carriers back flow to electrode, though it is difficult to understand why the carriers capable to go beyond the potential barrier area should come back to the electrode not being held by the energy barrier they have just passed through.

Thus, thermionic emission prevails at the high temperatures and relatively low electric fields. Current caused by tunnel emission takes place at low temperatures and high values of electric fields.

Fig. 3 shows different types of carriers emission through the interface metal-polymer including hybrid models.

M. Abkowitz and others [11] developed an alternative model based on thermally activated tunneling where hopping movement of a carrier in dielectric is taken into account, but Coulomb potential and energy disorder of the system are disregarded.

They showed that experimental curves $J(F)$ could be specified by a reasonable combination of parameters. A more complicated version of this injection concept was presented using Monte-Carlo simulation methods by Garlstein and Conwell [12]. Their model takes into account energy disorders of any amorphous hopping systems and image forces potential. It allows one to evaluate the probability 


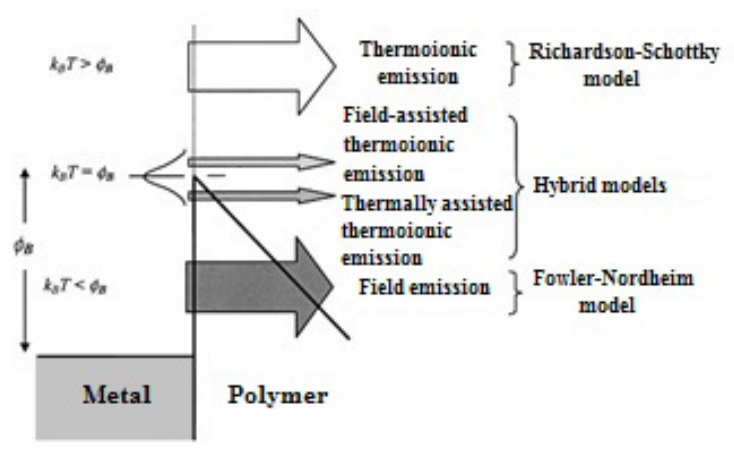

Fig. 3. Different types of emission of carriers through interface metalpolymer.

for the carrier to avoid a pair recombination with carrier image excluding the initial injection process. The complete description of the field and temperature dependence for injection current should evidently study the field and temperature dependences of primary injection process. It is necessary to study the following arbitrary motion of carriers taking into account the external field and images forces taking due care because of specific features of charge carrier motion in arbitrary media.

This problem was analyzed by Arkhipov and others [13] and dependence of injection current on electric field value, temperature and adherence of two hopping centers was obtained.

\section{Hopping transport of charge carriers}

Due to weak intermolecular binding, valence and conduction bands of molecular crystals are narrow, usually $0.1 \mathrm{eV}$ or less. As a result, the mean free path of a charge carrier between two consecutive acts of phonon scattering has an order of the crystal lattice constant, at least, at the ambient temperature.

Taking into account disorder present in amorphous organic solids, e.g. in polymers, it is necessary to admit that elementary phenomenon of transport in these systems is a hop of a charge carrier between adjacent transport molecules or segments of the main element in polymer chain hereinafter called transport centers.

In chemical terms, this is a reduction-oxidation process including chemically identical but physically different components. Dependence of charge carrier mobility on temperature and electric field should reflect the dependence of this elementary transfer on $T$ and $F$. Its activation energy will be a sum of intermolecular and intramolecular contributions. It is a result of physical nonequivalence of hopping centers due to local disorder and it is a specific feature of any amorphous organic solid. The latter occurs due to changes of molecular surroundings by adding / deletion of an electron to the transport center. A charge transfer requires concurrent molecular deformation, i.e. polaron transfer. Considerable difference between transfer models is related to a relative value of both contributions.

The hopping model supposes a weak connection between a charge carrier and intermolecular and intramolecular modes, activation energy of transfer reflects statistic energy disorder of the hopping centers. From the other hand, polaron model considers disorder energy to be insignificant as compared with molecular deformation energy.

Usually movement of the carriers in amorphous polymers is an activated process. As transfer time can't be measured accurately or even distinguished, the direct measurements of mobility are not possible.

To understand the process of macroscopic charge transfer in these substances it seems to be useful to study and classify the main transfer mechanisms of activation character.

Dispersion jumps [14-15]. This process is a tunneling of the carriers between two appropriate points and its probability depends on overlap of wave functions of initial and final states.

Activation energy origin is connected with some potential barrier between these two states, this barrier is to be overpassed by the carrier while jumping. This process is similar to transfer of small radius polarons with phonons.

Trap-controlled jumps [16]. In this process a carrier during its chaotic walk meets the trap and remains inside until it gets enough activation energy to be released. Energy should exceed the value required for small radius polarons jumps, total activation energy of transfer should include energy to escape from the trap and jump energy.

Multiple trapping [17]. Conductive area and trapping centers are necessary conditions for this process. If these centers are located at different depth, transfer is determined by the carriers trapping and releasing kinetics, while free carriers movement is typical for zone mechanism. The simplest type of hopping conductivity is realized at transfers between the nearest adjacent centers. Density state in zone of localized levels is presented by the curve with a maximum at energy $\varepsilon_{m}$. Most probably, initial and final jump points have the energy close to maximum of state density providing they are the nearest neighbors. To realize the jump the final point should be free. The probability of this event depends on distance to Fermi-level and proportional to [18]

$$
\exp \left(-\frac{\left|\varepsilon_{\mathrm{F}}-\varepsilon_{\mathrm{m}}\right|}{\mathrm{kT}}\right)
$$

Taking into account the factor (3), a complete expression of specific conductivity considering percolation radius $r_{c}$ in the system of random points with $N$ concentration can be written as:

$$
\sigma(\mathrm{T})=\sigma_{0} \exp \left(-\frac{1.73}{\mathrm{~N}^{1 / 3} \mathrm{a}}\right) \exp \left(-\frac{\left|\varepsilon_{\mathrm{F}}-\varepsilon_{\mathrm{m}}\right|}{\mathrm{kT}}\right) .
$$

To realize the conductivity by jumps to the nearest centers it is necessary to have many pairs of the nearest neighbours where one center is free. If the temperature is decreased to $k T<<\left|\varepsilon_{F}-\varepsilon_{m}\right|$, there will be too little free places among the nearest neighbours, the major part of which have $\varepsilon_{m}$, energy and jumps to the nearest centers will stop. Then jumps between centers with some $\mathcal{\varepsilon}$-region energy of Fermi level with definite availability of free places should become considerable. The issue is how close these centers are to each other. In this case the conductivity is as follows:

$$
\sigma=\sigma_{0} \exp \left(-\frac{\mathrm{T}_{\mathrm{M}}}{\mathrm{T}}\right)^{1 / 4} \text {. }
$$


Value $\sigma_{0}$ depends on temperature by power law, but usually this dependence is disregarded.

As average jump length $\langle r\rangle$ depends on $T$, it is possible to speak about hopping conductivity with a variable length of jump. The power $1 \frac{1}{4}$ in exponent (6) can be only in threedimensional dielectric [19]. For a thin film a reduction of dimensionality of system down to two occurs that is why the resistance and temperature relation now has the following form [20]:

$$
\sigma=\sigma_{0} \exp \left(-\frac{T_{M}}{T}\right)^{1 / 3}
$$

\section{Hopping transport with Gaussian distribution of energy levels}

In the works [21-23] the following model of the charge carrier transfer is developed in details. Organic matrix is modeled by a cubic lattice with an edge $a$ in each site of which a transport center is located. Position of energy level $\varepsilon$, which takes a part in charge transport is an independent random value due to a random non-correlated influence of surroundings. The relevant state density is described by a Gaussian distribution:

$$
\rho(\varepsilon)=\left(2 \pi \sigma^{2}\right)^{-1 / 2} \cdot \exp \left(-\varepsilon^{2} / 2 \sigma^{2}\right) .
$$

This choice of $\rho(\varepsilon)$ is explained by an experimentally found Gaussian form of absorption bands and fluorescence of many polymers. It means that the total energy of interaction between center and surroundings is a sum of a big number of independent items, probabilities densities of which do not differ considerably. The equation suggested by Miller and Abrahams [24] is chosen for jumps frequency between centers $i$ and $j$ :

$$
\mathrm{v}_{\mathrm{ij}}=\mathrm{v}_{0} \exp \left(-2 \gamma \mathrm{r}_{\mathrm{ij}}\right) \exp \left(-\frac{\left|\varepsilon_{\mathrm{j}}-\varepsilon_{\mathrm{i}}\right|+\left(\varepsilon_{\mathrm{j}}-\varepsilon_{\mathrm{i}}\right)}{2 \mathrm{kT}}\right),
$$

where $v_{0}$ is a pre-exponential frequency factor, $\gamma$ is a wave function damping coefficient, $r_{i j}$ is a distance between centers equal according to the subject model to $a$.

Function $\rho(\varepsilon)$ describes the effects of energy disorder. Geometrical disorder can be described phenomenologically assuming $y a$ a random value. Concrete calculations within the frames of the described model were successfully carried out only with a help of numerical simulations by MonteCarlo method. The field dependence of mobility has an Sshape character, its medium part approximately corresponds to a linear dependence of $\ln \mu$ on $F^{1 / 2}$. However, this region is considerably narrower than that in the experiment. Taking into account the geometric disorder within the frames of the described model results in interesting consequences mobility becomes a nonmonotonic function of the field, initially decreasing and then increasing with the field increase [23]. This behavior well corresponds to the one observed in the experiments of Borsenberger [25] and Abkowitz [26].

\section{Polaron transport}

Interaction between electrons and ions of lattice is an electrostatic one, it causes local deformation of a lattice. The electron is accompanied by this deformation during its move across the lattice. A combination of an electron and a stress field of the lattice created by it is called a polaron. There are big and small polarons. The electrons of big polarons move in energy zone but they have enlarged mass; these are the polarons which have been studied above. An electron of a small polaron [27] spends the majority of time in a bound state captured by some separate ion. At a high temperature this electron tunnels slowly through a crystal as if it were in a part of energy zone relevant to a big effective mass.

The works of Schein and his colleagues [28, 29] develop an idea that polarons of a small radius are the charge carriers in disordered organic matrixes. In other words, polaron is a bound state of a charge carrier and vibrational mode (intramolecular or phonons) which moves in a solid as a single whole. The above mentioned works consider the polaron to be formed when a carrier interacts with intramolecular vibrational mode. Within the weak external field the polaron mobility can be written as follows:

$$
\mu=\frac{e \rho^{2}}{\mathrm{~T}} \mathrm{P} \frac{\omega}{2 \pi} \exp \left(-\frac{1 / 2 \mathrm{E}_{\mathrm{p}}-\mathrm{J}}{\mathrm{T}}\right),
$$

where $P$ is a probability of a charge carrier transfer from point to point when energy levels at these points are coincided, $\omega$ is a frequency of vibrational mode, $E_{p}$ polaron binding energy, $\rho$ is an average distance between points (transport centers), $J$ is a two-center resonance integral. Function $P$ is complicated but it can be written rather simply in two extreme cases - big and small $J$.

In the first (adiabatic) regime $P=1$, in the second (nonadiabatic) regime $P \propto J^{2} \propto \exp (-2 \gamma \rho)$. As it was mentioned in the work [28], from the expression (9) it follows that in the first case activation energy isincreased with an average distance between centers (as $J$ is decreased), in the second case it doesn't depend on distance ( $J$ is insignificant).

Importance of polaron effects for the charge carriers transfer in organic amorphous materials is actively discussed, because the purely polaron model is not able to describe charge transfer due to the large values of such parameters as polaron activation energy $E_{a}=E_{p} / 2$ and transfer integral $J$. However, for some organic systems deformation energy can be compared with disorder energy, therefore, description of charge carriers transfer in such materials should take into account superposition of disorder influence and polaron effects.

In case of polaron hopping transport in nonadiabatic approximation the jump speed of a small radius polaron is given according to Marcus model $[30,31]$ :

$$
v_{i j}=\frac{J^{2}}{\hbar} \sqrt{\frac{\pi}{4 E_{a} k T}} \exp \left(-\frac{E_{a}}{k T}\right) \exp \left[-\frac{E_{j}-E_{i}}{2 k T}-\frac{\left(E_{j}-E_{i}\right)^{2}}{16 E_{a} k T}\right] .
$$

Recently the authors of [32] formulated a theory of effective medium approximation (EMA) to describe polaron transfer in amorphous organic system using (10). After taking into account the correlation effect the result corresponds to the Poole-Frenkel dependence $\ln \mu \propto \sqrt{\mathrm{F}}$. The presented theory suggests the test which can be used to differentiate polaron transport from non polaron one [32]. It appears that the dependence of mobility on the electric field changes from a nearly linear one to $T^{-3 / 2}$ in the case of the non polaron 
transport, when Miller-Abrahams formalism is applicable, and deviates considerably from the linearity to $T^{3 / 2}$ for the polaron transport. Therefore, the existence of such deviations proves the necessity to account for polarons in the studied systems.

Besides, the presented model of polarons can explain quantitatively the observed dependencies of polaron mobility on temperature and field suggesting reasonable values for polaron binding energy and transfer integral. It is important that field dependence of Poole-Frenkel $\ln \mu \propto \sqrt{\mathrm{F}}$ takes place for both the pure charge transport and polaron one providing that energy correlation effects are taken into account. Temperature dependence of drift mobility is super-Arrhenius one, $\ln \mu \propto 1 / \mathrm{T}^{2}$, and can be observed for polaron transport providing that activation energy of polaron is relatively small. The results of presented EMA theory are in a good agreement with experimental results obtained for some $\sigma$-conjugated polysilanes where the importance of polaron effects is firmly established.

Is it possible to state that charge transport in disordered organic solids is driven by disorder or small radius polarons $[33,34]$ ? No doubt, a moving charge carrier always causes structure deformations of medium. The question concerns the quanitity of the effect.

The model based only on polaron effects is not capable to describe the charge transport as required parameters appear physically not reasonable. In adiabatic approximation activation energy for polaron transport $\Delta=E_{p} / 2-J$, where $J$ is electron integral of transfer [35]. Therefore, if $\Delta$ is changed between 0.3 and $0.6 \mathrm{eV}$, then $E_{p} \approx 0.6-1.2 \mathrm{eV}$, what is inacceptably big and contradicts both quantum chemical calculations [36] and theoretical studies of charge transport in molecular crystals.

In the final part of the work we'll consider the results of studies of the mechanisms of the charge carriers transfer in thin films of polydiphenylenephthalide (PDPh) from the class of polyarylenephthalides. One of the interesting PDPh film properties is a transfer from dielectric state to highly conductive state (HCS).A possible explanation of the transfer to HCS can be an appearance of a narrow zone near the middle of a band gap of the polimer through which a coherent charge carrier occurs. A probability of such a zone appearance is proved by the results of [37-39]. Registration of negative differential resistance of $N$-type [40] is explained by the presence of such a zone.

In PDPh films with a wide band gap a low-barrier Schottky effect can be observed [41]. This effect is explained by a barrier which forms at the interface metal-polymer, so that its height $\Delta \varphi$ is determined by a difference between the energy of Fermi-level of contacting electrode and energy of trapping level formed near the mid of polymer band gap. In [42-43] the peculiarities of voltage-current characteristics are studied within the frames of injection theory of currents limited by spatial charge. Nanostructured heterointerfaces created on the base of silicon and PDPh films are shown to be rather promising to control output value of current.

Peculiarities of charge transfer in film samples with a structure semi-conductor/PDPh/metal are explained within the frames of three models: hopping transport on trapping levels, Schottky emission and field tunnel emission [44].

Time-of-flight method showed that dispersion transport takes place in film samples with a thickness exceeding critical one, and when mechanic pressure is applied charge carriers are not changed as it occurs at thickness less or close to critical one, holes remain the main charge carriers [45-47].

The principles of formation of nanostructured organicorganic interface with increased conductivity at two polymer films interface are described in [48-51]. It is established that conductivity is determined by the type and degree of external influence. It is shown that development of chemical sensors, e.g., relative humidity sensors, ethyl alcohol vapors pressure sensors and $\mathrm{pH}$ indicators is possible on the base of this interface [52].

Acknowledgements. The work was supported by the grant of the Russian Foundation for Basic Research 14-02-970008_r_ povolzhie_a.

\section{References}

1. G. Hadziioannou, P. F. Hutten. Semiconducting Polymers. Chemistry, Physics and Engineering. WILEY-VCH Verlag GmbH (2000).

2. R. Schmechel and H. Seggern. Phys. stat. sol. (a), 201, 1215 (2004).

3. D. Monro. Phys. Rev. Lett., 54, 146 (1985).

4. S.D. Baranovskii, H. Cordes, F. Hensel, G. Leising. Phys. Rev. B, 62, 7934 (2001).

5. V.I. Arkhipov, E.V. Emelianova, G. J. Adriaenssens, H. Bassler. J. Non-Cryst. Solids, 299-302, 1047 (2001).

6. S. M. Sze. Physics of semiconductor devices, J. Wiley \& Sons (1981).

7. V.L. Rideout. Thin Solid Films, 48, 261 (1978).

8. R.H. Fowler, L. Nordheim. Proc. R. Soc. Lond. A, 119, 173 (1928).

9. I. D. Parker. J. Appl Phys., 75, 1656 (1994).

10. P. S. Davids, Sh. M. Kogan, I. D. Parker, D. L. Smith. Appl. Phys. Lett., 69, 2270 (1996).

11. M. A. Abkowitz, H. A. Mizes, J. S. Facci. Appl. Phys. Lett., 66, 1288 (1995).

12. Yu. N. Garlstein, E. M. Conwell. Chem. Phys. Lett., 255, 93 (1996).

13. V.I. Arkhipov, E. V. Emelianova, Y.H. Tak, H. Bassler. J. Appl. Phys., 84, 848 (1998).

14. G. Pfister, C. H. Grffiths. Phys. Rev. Lett., 40, 659 (1978).

15. H. Scher, E. W. Montroll. Phys. Rev. B, 12, 2455 (1975).

16. G. Pfister, S. Grammatica, J. Mort. Phys. Rev. Lett., 37, 1360 (1976).

17. M. Silver, L. Cohen. Phys. Rev. B, 15, 3276 (1977).

18. B. I. Shklovskii, A. L. Efros. Electronic properties of doped semiconductors. Springer-Verlag (1984).

19. N. F. Mott. J. Non-Cryst. Sol., 1,1 (1968).

20. E. Arnold. Appl. Phys. Lett., 25, 705 (1974).

21. M. Hori, F. Yonezawa. J. Phys. C, 10, 229 (1977).

22. L. Pautmeier, R. Richert, H. Bassler. Philos. Mag. B, 63, 587 (1991).

23. R. Richert, L. Pautmeier, H. Bassler. Phys. Rev. Lett., 63, 547 (1989).

24. A. Miller, E. Abrahams. Phys. Rev., 120, 745 (1960).

25. P. M. Borsenberger, L. Pautmeier, H. Bassler. J. Chem. 
Phys., 94, 5447 (1991).

26. M. A. Abkowitz. Philos. Mag. B, 65, 817 (1992).

27. T. Holstein. Ann. of Phys., 8, 343 (1959).

28. J. X. Mack, L. B. Schein. A. Peled. Phys. Rev. B, 39, 7500 (1989).

29. L. B. Schein, D. Glatz, J. C. Scott. Phys. Rev. Lett., 65, 472 (1990).

30. R. A. Marcus. J. Chem. Phys., 24, 966 (1956).

31. R. A. Marcus. Rev. Mod. Phys., 65, 599 (1993).

32. I. I. Fishchuk, A. Kadashchuk, H. Bassler, S. Nespurek. Phys. Rev. B, 67, 224303 (2003).

33. P.E. Parris, V.M. Kenkre, D.H. Dunlap. Phys. Rev. Lett., 87, 126601 (2001).

34. L. B. Schein. Philos. Mag. B, 65, 795 (1992).

35. D. Emin. Phys. Rev. B, 46, 9419 (1992).

36. Z. Shuai, J. L. Bredas, W. P. Su. Chem. Phys. Lett., 228, 301 (1994).

37. A.A. Bunakov, A. N. Lachinov, R. B. Salikhov. Technical Physics, 48 (5), 626 (2003).

38. R. B. Salikhov, A. N. Lachinov, A. A. Bunakov. Phys. of the Solid State, 49 (1), 185 (2007).

39. R. B. Salikhov, A.N. Lachinov, R.G. Rakhmeyev. Molecular Crystals and Liquid Crystals. 467 (1), 85 (2007).

40. A.A. Bunakov, A.N. Lachinov, R. B. Salikhov. Butlerovskiye soobtcheniya, 3 (11), 64 (2002) (in Russian) [Бунаков А.А., Лачинов А.Н., Салихов Р.Б. Бутлеровские сообщения, 3 (11), 64 (2002)].

41. R.B. Salikhov, A.N. Lachinov, R.G. Rakhmeev. Semiconductors, 41 (10), 1165 (2007).

42. A. A. Bunakov, A. N. Lachinov, R. B. Salikhov. Macromol. Symposia, 212, 387 (2004).
43. R.B. Salikhov, A.N. Lachinov, A.A. Bunakov. Nanotekhnika, 2, 43 (2008) (in Russian) [Салихов Р. Б., Лачинов А.Н., Бунаков А.А. Нанотехника, 2, 43 (2008)].

44. R. B. Salikhov, A. N. Lachinov, R. G. Rakhmeev. J. Appl. Phys., 101, 053706 (2007).

45. A.N. Lachinov, R. B Salikhov, A.A. Bunakov., A. R. Tameev. Nonlinear Optics, Quantum Optics, 32, 13 (2004).

46. A.R. Tameev, A.N. Lachinov, R.B. Salikhov, A.A. Bunakov, A.V. Vannikov. Russian Journal of Physical Chemistry, 79 (12), 2025 (2005).

47. A.R. Tameev, A.V. Vannikov, R.G. Rakhmeev, A.N. Lachinov, V.R. Nikitenko, R.B. Salikhov, A.A. Bunakov. Physics of the Solid State, 53 (1), 195 (2011).

48. R. B. Salikhov, A. N. Lachinov, R. G. Rakhmeev. Technical Physics Letters, 34 (6), 495 (2008).

49. R.B. Salikhov, A.N. Lachinov, V.M. Kornilov, R. G. Rakhmeev. Technical Physics, 54 (4), 575 (2009).

50. R.M. Gadiev, A.N. Lachinov, V.M. Kornilov, R. B. Salikhov, R. G. Rakhmeev, A. R. Yusupov. Pis'ma v JETF, 90 (11), 821 - 825 (2009) (in Russian) [Гадиев Р. M., Лачинов А.Н., Корнилов В.М., Салихов Р.Б., Рахмеев Р.Г., Юсупов А.Р. Письма в ЖЭТФ, 90 (11), $821-825$ (2009)].

51. R.B. Salikhov, A.N. Lachinov, R.G. Rakhmeev, R.M. Gadiev. Molecular Crystals and Liquid Crystals, 535, 74 (2011).

52. R.B. Salikhov, A.N. Lachinov, R.G. Rakhmeev, R. M. Gadiev, A. R. Yusupov, S. N. Salazkin. Measurement Techniques, 52 (4), 427 (2009). 